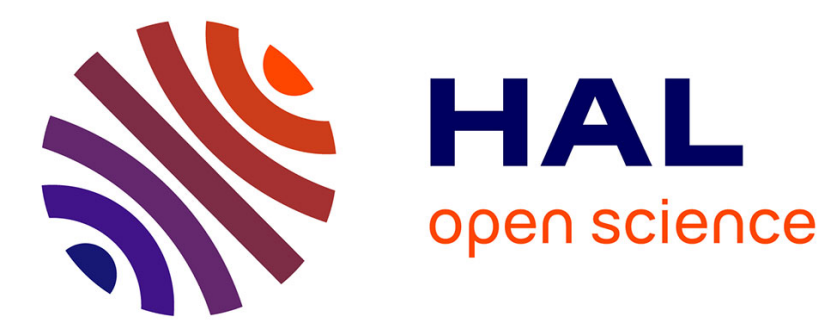

\title{
Reduced ODE dynamics as formal relativistic asymptotics of a Peierls-Nabarro model
}

\author{
Hassan Ibrahim, Régis Monneau
}

\section{To cite this version:}

Hassan Ibrahim, Régis Monneau. Reduced ODE dynamics as formal relativistic asymptotics of a Peierls-Nabarro model. 2013. hal-00789494

\section{HAL Id: hal-00789494 https://hal.science/hal-00789494}

Preprint submitted on 18 Feb 2013

HAL is a multi-disciplinary open access archive for the deposit and dissemination of scientific research documents, whether they are published or not. The documents may come from teaching and research institutions in France or abroad, or from public or private research centers.
L'archive ouverte pluridisciplinaire HAL, est destinée au dépôt et à la diffusion de documents scientifiques de niveau recherche, publiés ou non, émanant des établissements d'enseignement et de recherche français ou étrangers, des laboratoires publics ou privés. 


\title{
Reduced ODE dynamics as formal relativistic asymptotics of a Peierls-Nabarro model
}

\author{
H. Ibrahim ${ }^{\dagger, \ddagger}$, R. Monneau *
}

February 18, 2013

\begin{abstract}
In this paper, we consider a scalar Peierls-Nabarro model describing the motion of dislocations in the plane $\left(x_{1}, x_{2}\right)$, along the line $x_{2}=0$. Each dislocation can be seen as a phase transition and creates a scalar displacement field in the plane. This displacement field solves a simplified elasto-dynamics equation which is simply the linear wave equation. The total displacement field creates a stress which makes move the dislocation themselves. By symmetry, we can reduce the system to the wave equation in the half plane $x_{2}>0$ coupled with an equation for the dynamics of dislocations on the boundary of the half plane, i.e. on $x_{2}=0$. Our goal is to understand the dynamics of well-separated dislocations in the limit when the distance between dislocations is very large of order $1 / \varepsilon$. After rescaling, this corresponds to introduce a small parameter $\varepsilon$ in the system. In the limit $\varepsilon \rightarrow 0$, we are formally able to identify a reduced ODE model describing the dynamics of relativistic dislocations.
\end{abstract}

AMS subject classifications: 70H40, 45M05, 35D30

Key words: Peierls-Nabarro model, dislocation dynamics, relativitic dymamics, formal asymptotics, reduced model.

\section{Introduction}

\subsection{Setting of the problem}

In this paper, we consider a scalar Peiels-Nabarro model describing the dynamics of dislocations in the plane $\left(x_{1}, x_{2}\right)$, along the line $x_{2}=0$. This is a phase field model, where each dislocation can be seen as a phase transition essentially between two consecutive integers. We refer to [11] for an overview on the Peierls-Nabarro model. Our scalar Peiels-Nabarro model (see (1.1) below) can

\footnotetext{
*Université Paris-Est, Ecole des Ponts, CERMICS, 6 et 8 avenue Blaise Pascal, Cité Descartes Champs-surMarne, 77455 Marne-la-Vallée Cedex 2, France. E-mail: monneau@cermics.enpc.fr

$\dagger$ Lebanese University, Faculty of Sciences, Mathematics Department, Hadeth, Beirut, Lebanon.

${ }^{\ddagger}$ School of arts and sciences, Mathematics Department, Lebanese International University (LIU), Beirut Campus, Al-Mouseitbeh, P.B. Box 14-6404, Beirut, Lebanon. Email: hassan.ibrahim@liu.edu.lb
} 
be seen as a scalar simplification of the vectorial Peierls-Nabarro Galerkin model introduced in [2] (see also [3]).

A dislocation is a defect in a crystal and creates a stress field in the material (see [8]). The total stress field creates a force acting on each dislocation, and makes those dislocations move on the line $x_{2}=0$. The whole model can be seen as a coupling between a dynamics on the line $x_{2}=0$ and a dynamics outside the line $x_{2}=0$.

In the model that we consider, the phase field is a scalar quantity which can be identified to a scalar displacement of atoms in the crystal. This displacement satisfies a scalar elasto-dynamics equation, which is simply the linear wave equation in the plane, outside the line $x_{2}=0$. By symmetry, we can reduce the problem to the wave equation on the half plane $x_{2}>0$ coupled with a Peierls-Nabarro type dynamics on the boundary of the half plane, i.e. on $x_{2}=0$. We are interested in the dynamics of well-separated dislocations and in the limit when the distance between dislocations is very large, of order $1 / \varepsilon$. After a suitable rescaling, it corresponds to introduce a small parameter $\varepsilon>0$ in the model and then to study the limit $\varepsilon \rightarrow 0$. More precisely we consider a phase field $u^{\varepsilon}\left(x_{1}, x_{2}, t\right)$ which is a real function solution of the following system

$$
\begin{cases}\square u^{\epsilon}=0, & x_{2}>0 \\ N_{\varepsilon} u^{\varepsilon}=0, & x_{2}=0\end{cases}
$$

where the two operators $\square$ and $N_{\varepsilon}$ applied on a scalar function $u=u(x, t), x=\left(x_{1}, x_{2}\right) \in \mathbb{R}^{2}$ are defined as follows:

$$
\begin{cases}\square u:=\frac{1}{c_{0}^{2}} u_{t t}-\Delta u, & x_{2}>0, \\ N_{\varepsilon} u:=\beta\left(\frac{1}{c_{0}^{2}} u_{t t}-\partial_{11} u\right)+k u_{t}-\frac{1}{\varepsilon}\left(\partial_{2} u-\frac{1}{\varepsilon} W^{\prime}(u)+\sigma\left(x_{1}, t\right)\right), & x_{2}=0 \\ \beta=m c_{0}^{2}, & \end{cases}
$$

where $c_{0} \in(0, \infty)$ is the velocity of sound in the crystal, and $m, k \in[0, \infty)$ are parameters. The quantity $m$ can be interpreted as a kind of mass of the dislocation and $k$ can be seen as a damping factor that is classical for the evolution Peierls-Nabarro model (see for instance [7]). Here we use the notation $u_{t}=\frac{\partial u}{\partial t}, u_{t t}=\frac{\partial^{2} u}{\partial t^{2}}, \partial_{i} u=\frac{\partial u}{\partial x_{i}}, \partial_{i i} u=\frac{\partial^{2} u}{\partial x_{i}^{2}}$ for $i=1,2$ and $\Delta u=\partial_{11} u+\partial_{22} u$. In this model, the scalar-valued function $W$ is a 1-periodic smooth potential mimicking the periodicity of the atoms in the crystal. We assume that $W$ satisfies

$$
\left\{\begin{array}{l}
W(u+1)=W(u) \text { for any } u \in \mathbb{R} \\
W=0 \text { on } \mathbb{Z} \\
W>0 \text { on } \mathbb{R} \backslash \mathbb{Z} \\
\alpha_{0}:=W^{\prime \prime}(0)>0 .
\end{array}\right.
$$

A dislocation will be naturally seen as a phase transition between a two consecutive minima of $W$. In this model, we consider the presence of a given exterior scalar stress field $\sigma\left(x_{1}, t\right)$ which has a contribution to the force acting on the dislocations on $x_{2}=0$. This contribution is taken into 
account in the definition of the operator $N_{\varepsilon}$. The limit $\varepsilon \rightarrow 0$ has been studied rigorously in [7] in the particular case $\beta=0, c_{0}=+\infty$ and $k=1$, which corresponds to a quasistatic approximation. In the present paper our goal is to study formally the limit $\varepsilon \rightarrow 0$ for our more general model in the relativistic regime.

For $\sigma \equiv 0$, the simplest situation is the case of a single and stationary dislocation

$$
u^{\varepsilon}(x, t)=\phi\left(\frac{x}{\varepsilon}\right)
$$

where $\phi$ is a normalized phase transition between 0 and 1 , solution of the following system:

$$
\begin{cases}\Delta \phi=0, & x_{2}>0 \\ \beta \partial_{11} \phi+\partial_{2} \phi-W^{\prime}(\phi)=0, & x_{2}=0 \\ \phi(-\infty, 0)=0, \quad \phi(+\infty, 0)=1 . & \end{cases}
$$

We are interested in the dynamics of $N \geq 1$ dislocations of positions $X_{i}(t) \in \mathbb{R}$ for $i=1, \ldots, N$ on the axis $x_{2}=0$. Because we are considering a relativistic regime, it is natural to introduce the following relativistic coefficient:

$$
\gamma_{i}(t)=\frac{1}{\sqrt{1-\left(\frac{X_{i}^{\prime}(t)}{c_{0}}\right)^{2}}}
$$

where ()$^{\prime}$ denotes the time derivative. This coefficient $\gamma_{i}$ encodes the contraction of the fields in the $x_{1}$ direction. Then a natural ansatz for describing the phase transition associated to those dislocations is the following

$$
\hat{u}^{\varepsilon}(x, t)=\left\{\sum_{i=1}^{N} \phi\left(\gamma_{i}(t)\left(\frac{x_{1}-X_{i}(t)}{\varepsilon}\right), \frac{x_{2}}{\varepsilon}\right)\right\}+\varepsilon v^{\varepsilon}(x, t)
$$

where $v^{\varepsilon}$ appears to be a correction term that will be precised later. Such an ansatz is compatible with the dynamics (1.1) only for suitable correction terms $v^{\varepsilon}$ which impose the following asymptotical dynamics:

$$
m_{0}\left(\gamma_{i} X_{i}^{\prime}\right)^{\prime}+k_{0} \gamma_{i} X_{i}^{\prime}=-\sigma\left(X_{i}, t\right)+\frac{1}{\pi} \sum_{j \neq i} \frac{1}{\gamma_{j}} \frac{1}{\left(X_{i}-X_{j}\right)}, \quad i=1, \ldots, N
$$

where $m_{0}, k_{0}$ are parameters that will be precised later. Our ODE dynamics (1.6) is similar to equation (1) in [15].

The term $\left(\gamma_{i} X_{i}^{\prime}\right)^{\prime}=\gamma_{i}^{3} X_{i}^{\prime \prime}$ is the natural relativistic acceleration and $m_{0} \gamma_{i}^{3}$ is the effective mass of the dislocation, which is coherent with the one computed in [9] for screw dislocations (see also (3.12) in [13]).

The term $k_{0} \gamma_{i} X_{i}^{\prime}$ can be seen as a friction term (viscous force) which will slow down the motion of the dislocations. This term is compatible with the one given in (2.21) and (2.18) in [13], for the Eshelby approximation [5]. We will see that the damping factor $k_{0}$ vanishes when the coefficient $k$ vanishes in (1.2). The precise statement of our result is given in Theorem 1.1. 


\subsection{Assumptions}

We will choose

$$
v^{\varepsilon}(x, t)=\frac{\sigma\left(x_{1}, t\right)}{W^{\prime \prime}(0)}-\sum_{i=1}^{N} \sum_{\alpha=1,2,3} a_{i}^{\alpha}(t) \psi^{\alpha}\left(\gamma_{i}(t)\left(\frac{x_{1}-X_{i}(t)}{\varepsilon}\right), \frac{x_{2}}{\varepsilon}\right)
$$

where the coefficients $a_{i}^{\alpha}$ are given by

$$
a_{i}^{1}:=\frac{2 \gamma_{i}^{\prime} X_{i}^{\prime}}{c_{0}^{2}}, \quad a_{i}^{2}:=\frac{\gamma_{i}^{\prime} X_{i}^{\prime}+\left(\gamma_{i} X_{i}^{\prime}\right)^{\prime}}{c_{0}^{2}} \quad \text { and } \quad a_{i}^{3}:=k \gamma_{i} X_{i}^{\prime} \quad \text { for } \quad i=1, \ldots, N \text {. }
$$

Here we assume the existence of corrector functions $\psi^{\alpha}, \alpha=1,2,3$ which satisfy:

$$
\begin{gathered}
\begin{cases}\Delta \psi^{1}=x_{1} \partial_{11} \phi, & x_{2}>0 \\
A \psi^{1}=\beta x_{1} \partial_{11} \phi-\frac{\bar{\beta}_{0}}{2 \alpha_{0}}\left(W^{\prime \prime}(\phi)-W^{\prime \prime}(0)\right), & x_{2}=0,\end{cases} \\
\begin{cases}\Delta \psi^{2}=\partial_{1} \phi, & x_{2}>0 \\
A \psi^{2}=\beta \partial_{1} \phi+\frac{\bar{\beta}_{0}}{\alpha_{0}}\left(W^{\prime \prime}(\phi)-W^{\prime \prime}(0)\right), & x_{2}=0,\end{cases}
\end{gathered}
$$

and

$$
\begin{cases}\Delta \psi^{3}=0, & x_{2}>0 \\ A \psi^{3}=\partial_{1} \phi+\frac{\bar{k}_{0}}{\alpha_{0}}\left(W^{\prime \prime}(\phi)-W^{\prime \prime}(0)\right), & x_{2}=0,\end{cases}
$$

where the linearized operator $A$ is defined by:

$$
A \psi:=\beta \partial_{11} \psi+\partial_{2} \psi-W^{\prime \prime}(\phi) \psi
$$

It is possible to see (see Corollary 3.2) that those correctors can only exist if they satisfy a compatibility condition which forces the following values of the parameters:

$$
\bar{k}_{0}=\int_{\left\{x_{2}=0\right\}}\left(\partial_{1} \phi\right)^{2} \text { and } \bar{\beta}_{0}=\int_{\left\{x_{2}>0\right\}}\left(\partial_{1} \phi\right)^{2}+\int_{\left\{x_{2}=0\right\}} \beta\left(\partial_{1} \phi\right)^{2} .
$$

We also define the following parameters

$$
k_{0}=\bar{k}_{0} k \quad \text { and } \quad m_{0}=\frac{\bar{\beta}_{0}}{c_{0}^{2}}=\frac{1}{c_{0}^{2}} \int_{\left\{x_{2}>0\right\}}\left(\partial_{1} \phi\right)^{2}+m \int_{\left\{x_{2}=0\right\}}\left(\partial_{1} \phi\right)^{2},
$$

where $m_{0}$ can be interpreted as a kind of effective mass of the dislocation. For the simplicity of notation, we call $\psi^{0}=\phi$ and set the Heaviside function $H\left(x_{1}\right)=\mathbf{1}_{[0,+\infty)}\left(x_{1}\right)$. We consider the 
following assumptions on the profile functions $\psi^{\alpha}, \alpha=0,1,2,3$,

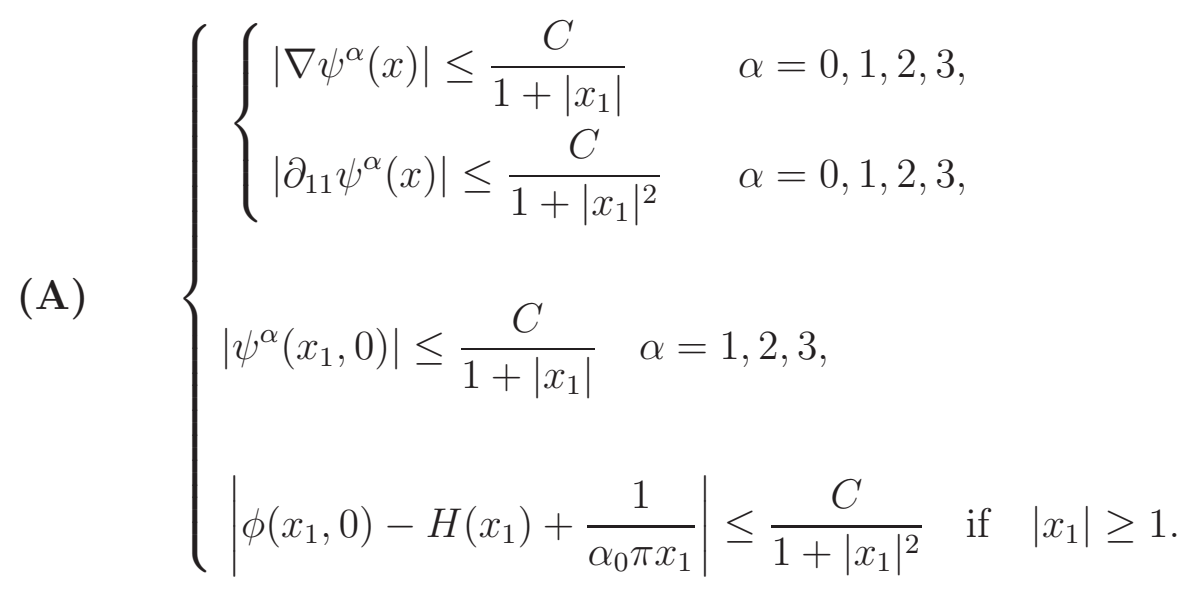

\subsection{The main result}

We show the following theorem:

\section{Theorem 1.1 (Reduced ODE dynamics as an asymptotic of the PN model)}

Let us consider the assumptions of Subsection 1.2, and assume $W$ and $\sigma$ smooth enough. For $i=1,2 \ldots, N$, let us consider particles $X_{i}=X_{i}(t)$ satisfying

$$
\left\{\begin{array}{c}
X_{i+1}(t)-X_{i}(t) \geq 2 \delta>0 \\
\left|X_{i}^{\prime}\right| \leq c_{0}(1-\delta), \quad \delta>0
\end{array} \mid \quad \text { for } \quad t \in[0, T]\right.
$$

solution of the dynamics (1.6) for $t \in(0, T)$, namely

$$
m_{0}\left(\gamma_{i} X_{i}^{\prime}\right)^{\prime}+k_{0} \gamma_{i} X_{i}^{\prime}=-\sigma\left(X_{i}, t\right)+\frac{1}{\pi} \sum_{j \neq i} \frac{1}{\gamma_{j}} \frac{1}{\left(X_{i}-X_{j}\right)}, \quad i=1, \ldots, N
$$

with $\gamma_{i}$ is given by (1.4) and the parameters $m_{0}, k_{0}$ are given in (1.13). Let us consider the ansatz function $\hat{u}^{\varepsilon}$ given by (1.5) and the correction term $v^{\varepsilon}$ given by (1.7) with the coefficients $a_{i}^{\alpha}$ given by (1.8). Then for any fixed $\delta>0$, we have as $\varepsilon \rightarrow 0$

$$
\begin{cases}\square \hat{u}^{\varepsilon}=O_{\delta}(1) & \text { uniformly in } L^{\infty}\left(\left\{x_{2}>0\right\} \times(0, T)\right) \\ N_{\varepsilon} \hat{u}^{\varepsilon}=O_{\delta}(1) & \text { uniformly in } L^{\infty}\left(\left\{x_{2}=0\right\} \times(0, T)\right) .\end{cases}
$$

Here we only get $O_{\delta}(1)$ right hand sides of (1.15). Nevertheless this still means that the ansatz (1.5) is a good approximation of the solution because the operator $N_{\varepsilon}$ involves $O\left(1 / \varepsilon^{2}\right)$-terms and the boundary condition involving $N_{\varepsilon}$ has consequences on the first PDE of (1.15). The important consequence of this result is the identification of the limit dynamics (1.6). We leave open the rigorous justification of this limit dynamics. The question of long time existence of solutions of the reduced ODE dynamics (1.6) is also open.

In the case of finite $\varepsilon$ for the Peierls-Nabarro Galerkin models, the effective dislocation dynamics can reveal retardation effects. We refer the reader for instance to $[12,14,16]$.

The interested reader can also consult [6] to see how the classical Peierls-Nabarro model can be rigorously derived from the Frenkel-Kontorova model at a smaller scale. We also refer the reader to $[4,10]$ for the relation between the Peierls-Nabarro model and other models at larger scales. 


\subsection{Organization of the paper}

In Section 2, we recall the physical derivation of our Peierls-Nabarro type model (1.1). In Section 3 , we give some simple properties on the correctors including compatibility conditions. In order to do the proof of our main result, we start with preliminary computations on our ansatz $\hat{u}^{\varepsilon}$ in Section 4. Finally in Section 5, we give the proof of our main result, namely Theorem 1.1.

\section{Physical derivation of the model}

We consider a two-dimensional crystal and call $U\left(x_{1}, x_{2}, t\right)$ the horizontal displacement (along the axis $x_{1}$ ) of the atoms. A natural action of the system without dislocations describing waves of velocity $c_{0}$, is the following

$$
\int_{\mathbb{R}^{2} \times(0, T)} \frac{1}{2}|\nabla U|^{2}-\frac{1}{2 c_{0}^{2}}\left(U_{t}\right)^{2}
$$

We now assume that dislocations are localized on the line $x_{2}=0$ and can only move along this line. We also assume the antisymmetry

$$
U\left(x_{1},-x_{2}, t\right)=-U\left(x_{1}, x_{2}, t\right)
$$

but allow a jump of $u$ when we cross the line $x_{2}=0$ :

$$
U\left(x_{1}, 0^{+}, t\right)-U\left(x_{1}, 0^{-}, t\right)=: \eta\left(x_{1}, t\right) .
$$

Then the function $\eta$ can describe a dislocation as a phase transition between two integers (if we normalise to one unit the Burgers vector which is here a scalar quantity because $U$ is itself scalar). Then the action (2.1) has to be modified as follows

$$
\mathcal{A}(U, \eta):=\int_{\mathbb{R}^{2} \times(0, T)}\left\{\frac{1}{2}\left|\nabla U-\eta e_{2} \delta_{0}\left(x_{2}\right)\right|^{2}-\frac{1}{2 c_{0}^{2}}\left(U_{t}\right)^{2}\right\}+\int_{\left\{x_{2}=0\right\} \times(0, T)} \bar{W}(\eta) .
$$

Here $\left(e_{1}, e_{2}\right)$ the standard orthonormal basis and we had to substract a Dirac mass in order to compensate the jump of $u$. The last integral is an energy term created by the misfit of the upper and lower half crystal created by the presence of the dislocation. In particular $W$ is 1-periodic, non negative and vanishes on integers (the case $\eta \in \mathbb{Z}$ corresponding to the case of no misfit in the crystal). Then the natural Peierls model is the following

$$
\left\{\begin{array}{l}
\mathcal{A}_{U}^{\prime}=0 \\
\bar{k} \eta_{t}=-\mathcal{A}_{\eta}^{\prime}
\end{array}\right.
$$

where the first line is the first variation of the action with respect to $u$, and the second line is the gradient flow evolution of the field $\eta$ where $\bar{k}$ is a damping factor. Then it is easy to check that

$$
u\left(x_{1}, x_{2}, t\right)=\left\{\begin{array}{lll}
2 U\left(x_{1}, x_{2}, t\right) & \text { if } \quad x_{2}>0 \\
\eta\left(x_{1}, t\right) & \text { if } \quad x_{2}=0
\end{array}\right.
$$


solves (1.1) for $\varepsilon=1, \sigma=0, \beta=0, \bar{k}=\frac{k}{4}$ and $\bar{W}=\frac{1}{4} W$. More generally, we recover (1.1) if we consider the general action

$$
\begin{aligned}
\mathcal{A}(U, \eta):= & \frac{1}{\varepsilon} \int_{\mathbb{R}^{2} \times(0, T)}\left\{\frac{1}{2}\left|\nabla U-\eta e_{2} \delta_{0}\left(x_{2}\right)\right|^{2}+\left(\nabla U-\eta e_{2} \delta_{0}\left(x_{2}\right)\right) \cdot \Sigma-\frac{1}{2 c_{0}^{2}}\left(U_{t}\right)^{2}\right\} \\
& +\int_{\left\{x_{2}=0\right\} \times(0, T)}\left\{\frac{1}{\varepsilon^{2}} \bar{W}(\eta)+\bar{\beta}\left(\left|\partial_{1} \eta\right|^{2}-\frac{1}{c_{0}^{2}}\left(\eta_{t}\right)^{2}\right)\right\}
\end{aligned}
$$

assuming that $\operatorname{div} \Sigma=0$ and $\sigma\left(x_{1}, t\right):=2 e_{2} \cdot \Sigma\left(x_{1}, 0, t\right)$ and $\bar{\beta}=\frac{\beta}{4}$. We have to emphasize the fact that model (2.2) in the special case $\bar{\beta}=0$, is a simplified scalar version of a more general model called the Peierls-Nabarro Galerkin model [2], where the displacement $U$ is vectorial.

\section{Some remarks on the correctors}

For the special case of $W^{\prime}(u)=-\frac{1}{2 \pi a} \sin \left\{2 \pi\left(u-\frac{1}{2}\right)\right\}$ for some $a>0$, we recall (see [7]) that the solution $\phi_{0}$ of $(1.3)$ for $\beta=0$ is

$$
\phi_{0}(x)=\frac{1}{\pi} \arctan \left(\frac{x_{1}}{x_{2}+a}\right)+\frac{1}{2} .
$$

We also refer to [1] for properties of $\phi_{0}$ in the case $\beta=0$ for more general potentials $W$. From expression (3.1), it can be seen that assumption (A) on the profile functions, sounds reasonable (even if we have no proof of it, and the goal of the present paper is not to prove any rigorous results in this direction).

Let us consider a function $\Psi$ solution of

$$
\begin{cases}\Delta \Psi=F & \text { on } \quad \Omega:=\left\{x_{2}>0\right\} \\ A \Psi=G & \text { on } \quad \partial \Omega=\left\{x_{2}=0\right\}\end{cases}
$$

\section{Lemma 3.1 (Compatibility condition)}

If $\Psi$ solves (3.2) with sufficient decay at infinity, then we have

$$
\int_{\Omega} F \zeta+\int_{\partial \Omega} G \zeta=0 \quad \text { with } \quad \zeta=\partial_{1} \phi
$$

where $\phi$ is the solution of (1.3).

Proof of Lemma 3.1

Step 1: self-adjoint property

For $(F, G)$ and $(\hat{F}, \hat{G})$, let us define the scalar product as

$$
\int_{\Omega} F \hat{F}+\int_{\partial \Omega} G \hat{G}
$$


Then a simple computation (by integration by parts) shows that the operator $\Psi \mapsto(\Delta \Psi, A \Psi)$ is self-adjoint for this scalar product, i.e. for any $\Psi, \Phi$, we have

$$
\int_{\Omega}(\Delta \Psi) \Phi+\int_{\partial \Omega}(A \Psi) \Phi=\int_{\Omega} \Psi(\Delta \Phi)+\int_{\partial \Omega} \Psi(A \Phi)
$$

\section{Step 2: consequence}

Because $\phi$ solves (1.3), we deduce that $\zeta=\partial_{1} \phi$ solves the linearized equation, i.e.

$$
\left\{\begin{array}{lll}
\Delta \zeta=0 & \text { on } & \Omega \\
A \zeta=0 & \text { on } & \partial \Omega
\end{array}\right.
$$

Using (3.4), this implies immediately (3.3).

\section{Corollary 3.2 (Values of the parameters for the correctors)}

If $\psi^{1}, \psi^{2}, \psi^{2}$ solve respectively (1.9), (1.10), (1.11), then the values of the parameters $\bar{k}_{0}$ and $\bar{\beta}_{0}$ are given by (1.12).

\section{Proof of Corollary 3.2}

Applying Lemma 3.1 for $\Psi=\psi^{1}$ and using equation (1.9), we get

$$
\begin{aligned}
0 & =\int_{\Omega} x_{1}\left(\partial_{11} \phi\right) \partial_{1} \phi+\int_{\partial \Omega}\left(\partial_{1} \phi\right)\left\{\beta x_{1} \partial_{11} \phi-\frac{\bar{\beta}_{0}}{2 \alpha_{0}}\left(W^{\prime \prime}(\phi)-W^{\prime \prime}(0)\right)\right\} \\
& =\int_{\Omega}-\frac{\left(\partial_{1} \phi\right)^{2}}{2}+\int_{\partial \Omega}-\frac{\beta\left(\partial_{1} \phi\right)^{2}}{2}+\frac{\bar{\beta}_{0}}{2}
\end{aligned}
$$

where we have used integration by parts and the fact that $\phi(-\infty, 0)=0, \phi(+\infty, 0)=1, W^{\prime}(0)=$ $W^{\prime}(1)$ and $\alpha_{0}=W^{\prime \prime}(0)$. This identifies the value of $\bar{\beta}_{0}$. The reasoning is similar when dealing with $\psi^{2}$ and $\psi^{3}$.

\section{Preliminary computations}

The goal of this section is to prove two technical results, namely Lemmata 4.1 and 4.2 , that will be used in the next section to do the proof of our main result.

In order to simplify the presention, we will use the following notations.

\section{Abridged notations:}

- $\xi_{i}^{1}=\gamma_{i}(t)\left(\frac{x_{1}-X_{i}(t)}{\varepsilon}\right)$

- $\xi_{i}^{2}=\frac{x_{2}}{\varepsilon}$,

- $\xi_{i}=\left(\xi_{i}^{1}, \xi_{i}^{2}\right)$,

- $\phi_{i}=\phi\left(\xi_{i}\right), \quad \psi_{i}^{\alpha}=\psi^{\alpha}\left(\xi_{i}\right), \quad \tilde{\phi}_{i}=\phi_{i}-H\left(\xi_{i}^{1}\right)$, 
- $\partial_{p} \phi_{i}=\left(\partial_{p} \phi\right)\left(\xi_{i}\right), \quad \partial_{p q} \phi_{i}=\left(\partial_{p q} \phi\right)\left(\xi_{i}\right), \quad p, q=1,2$,

- $\partial_{p} \psi_{i}^{\alpha}=\left(\partial_{p} \psi^{\alpha}\right)\left(\xi_{i}\right), \quad \partial_{p q} \psi_{i}^{\alpha}=\left(\partial_{p q} \psi^{\alpha}\right)\left(\xi_{i}\right), \quad p, q=1,2$,

- $\partial_{t} \psi_{i}^{\alpha}=\frac{d}{d t}\left[\psi^{\alpha}\left(\xi_{i}\right)\right], \quad \partial_{t t} \psi_{i}^{\alpha}=\frac{d^{2}}{d t^{2}}\left[\psi^{\alpha}\left(\xi_{i}\right)\right]$.

Remark that in regards of the above notations, the function $\hat{u}^{\varepsilon}$ can be simply written

$$
\hat{u}^{\varepsilon}=\sum_{i} \phi_{i}+\varepsilon\left\{\frac{\sigma}{\alpha_{0}}-\sum_{i=1, ., N} \sum_{\alpha=1,2,3} a_{i}^{\alpha} \psi_{i}^{\alpha}\right\} .
$$

Then we have the following result:

\section{Lemma 4.1 (Computation of $W^{\prime}\left(\hat{u}^{\varepsilon}\right)$ )}

Assume (1.14) for some $\delta>0$ and assume $W$ smooth enough. Given the point $\left(x_{1}, t\right) \in \mathbb{R} \times[0, T]$, there exists $i_{0}=i_{0}\left(x_{1}, t\right) \in\{1,2, \ldots, N\}$ such that we have with the previous abridged notations for $x_{2}=0$ and for $\varepsilon$ small enough (depending on $\delta$ ):

$W^{\prime}\left(\hat{u}^{\varepsilon}\left(x_{1}, 0, t\right)\right)=W^{\prime}\left(\tilde{\phi}_{i_{0}}\right)+\varepsilon W^{\prime \prime}\left(\tilde{\phi}_{i_{0}}\right)\left\{\frac{\sigma\left(x_{1}, t\right)}{\alpha_{0}}-\sum_{\alpha=1,2,3} a_{i_{0}}^{\alpha}(t) \psi_{i_{0}}^{\alpha}-\frac{1}{\varepsilon} \sum_{i \in\{1, \ldots, N\} \backslash\left\{i_{0}\right\}} \frac{1}{\alpha_{0} \pi \xi_{i}^{1}}\right\}+O_{\delta}\left(\varepsilon^{2}\right)$.

\section{Proof of Lemma 4.1}

Using the expression (4.3) of $\hat{u}^{\varepsilon}$ and the periodicity of $W^{\prime}$, we can write

$$
W^{\prime}\left(\hat{u}^{\varepsilon}\right)=W^{\prime}\left(\left(\sum_{i} \tilde{\phi}_{i}\right)+\varepsilon\left(\frac{\sigma}{\alpha_{0}}-\sum_{i} \sum_{\alpha} a_{i}^{\alpha} \psi_{i}^{\alpha}\right)\right) .
$$

We recall from (1.14) that the values of the $X_{i}(t)$ are well separated, i.e.

$$
X_{i+1}(t)-X_{i}(t) \geq 2 \delta>0 .
$$

Then there exists an index $i_{0}=i_{0}\left(x_{1} t\right)$ (possibly non unique) such that

$$
\left|x_{1}-X_{i_{0}}(t)\right|=\inf _{i}\left|x_{1}-X_{i}(t)\right| .
$$

Step 1: computations for $i \neq i_{0}$

We have $\left|x_{1}-X_{i}(t)\right| \geq \delta>0$, which implies $\left|\xi_{i}^{1}\right| \geq \frac{\delta}{\varepsilon}$, and we deduce from the last line of assumption $(\mathrm{A})$ that

$$
\phi_{i}-H\left(\xi_{i}^{1}\right)+\frac{1}{\alpha_{0} \pi \xi_{i}^{1}}=O\left(\frac{1}{1+(\delta / \varepsilon)^{2}}\right)=O_{\delta}\left(\varepsilon^{2}\right),
$$

which shows that

$$
\tilde{\phi}_{i}+\frac{1}{\alpha_{0} \pi \xi_{i}^{1}}=O_{\delta}\left(\varepsilon^{2}\right) .
$$


Similarly, from the third line of (A), we deduce for $\alpha=1,2,3$

$$
\left|\psi_{i}^{\alpha}\right| \leq \frac{C}{1+\left|\xi_{i}^{1}\right|} \leq \frac{C}{1+\delta / \varepsilon}=O_{\delta}(\varepsilon)
$$

\section{Step 2: conclusion}

From (4.3) and (4.4), we obtain

$$
\left(\sum_{i} \tilde{\phi}_{i}\right)+\varepsilon\left(\frac{\sigma}{\alpha_{0}}-\sum_{i} \sum_{\alpha} a_{i}^{\alpha} \psi_{i}^{\alpha}\right)=\tilde{\phi}_{i_{0}}+\varepsilon\left(\frac{\sigma}{\alpha_{0}}-\sum_{\alpha} a_{i_{0}}^{\alpha} \psi_{i_{0}}^{\alpha}\right)+O_{\delta}\left(\varepsilon^{2}\right)-\sum_{i \neq i_{0}} \frac{1}{\alpha_{0} \pi \xi_{i}^{1}},
$$

that yields

$$
W^{\prime}\left(\hat{u}^{\varepsilon}\right)=W^{\prime}\left(\tilde{\phi}_{i_{0}}\right)+W^{\prime \prime}\left(\tilde{\phi}_{i_{0}}\right)\left\{\varepsilon\left[\frac{\sigma}{\alpha_{0}}-\sum_{\alpha} a_{i_{0}}^{\alpha} \psi_{i_{0}}^{\alpha}\right]-\sum_{i \neq i_{0}} \frac{1}{\alpha_{0} \pi \xi_{i}^{1}}\right\}+O_{\delta}\left(\varepsilon^{2}\right),
$$

where we have used a second order expansion of $W^{\prime}$ and the fact that $\frac{1}{\alpha_{0} \pi \xi_{i}^{1}}=O_{\delta}(\varepsilon)$ for $i \neq i_{0}$. This is exactly (4.2).

We also have the following result:

\section{Lemma 4.2 (Derivatives of the profile functions)}

We recall that $\psi^{0}=\phi$, and for $\alpha=0,1,2,3$, we set

$$
\Psi_{i}^{\alpha}(x, t):=\psi^{\alpha}\left(\gamma_{i}(t)\left(\frac{x_{1}-X_{i}(t)}{\varepsilon}\right), \frac{x_{2}}{\varepsilon}\right) .
$$

Under the assumptions and notations of Lemma 4.1, the following holds for $(x, t) \in \mathbb{R} \times \mathbb{R}^{+} \times[0, T]$

$$
\begin{cases}\partial_{1} \Psi_{i_{0}}^{\alpha}=\frac{\gamma_{i_{0}}}{\varepsilon} \partial_{1} \psi_{i_{0}}^{\alpha} & \text { and } \quad \partial_{1} \Psi_{i}^{\alpha}=O_{\delta}(1) \text { if } i \neq i_{0}, \\ \partial_{2} \Psi_{i_{0}}^{\alpha}=\frac{1}{\varepsilon} \partial_{2} \psi_{i_{0}}^{\alpha} & \text { and } \partial_{2} \Psi_{i}^{\alpha}=O_{\delta}(1) \text { if } i \neq i_{0}, \\ \partial_{t} \Psi_{i_{0}}^{\alpha}=-\frac{\gamma_{i_{0}} X_{i_{0}}^{\prime}}{\varepsilon} \partial_{1} \psi_{i_{0}}^{\alpha}+O(1) & \text { and } \quad \partial_{t} \Psi_{i}^{\alpha}=O_{\delta}(1) \text { if } i \neq i_{0}, \\ \partial_{11} \Psi_{i_{0}}^{\alpha}=\frac{\gamma_{i_{0}}^{2}}{\varepsilon^{2}} \partial_{11} \psi_{i_{0}}^{\alpha} & \text { and } \quad \partial_{11} \Psi_{i}^{\alpha}=O_{\delta}(1) \text { if } i \neq i_{0},\end{cases}
$$

and for all $i=1, \ldots, N$

$$
\left\{\begin{array}{l}
\partial_{22} \Psi_{i}^{\alpha}=\frac{1}{\varepsilon^{2}} \partial_{22} \psi_{i}^{\alpha} \\
\partial_{t t} \Psi_{i}^{\alpha}=\frac{1}{\varepsilon^{2}}\left(\gamma_{i} X_{i}^{\prime}\right)^{2} \partial_{11} \psi_{i}^{\alpha}+\frac{J_{i}^{\alpha}}{\varepsilon}+O(1) \quad \text { with } \quad J_{i}^{\alpha}:=-2 \gamma_{i}^{\prime} X_{i}^{\prime} \xi_{i}^{1} \partial_{11} \psi_{i}^{\alpha}-\left(\gamma_{i}^{\prime} X_{i}^{\prime}+\left(\gamma_{i} X_{i}^{\prime}\right)^{\prime}\right) \partial_{1} \psi_{i}^{\alpha}
\end{array}\right.
$$

with

$$
J_{i_{0}}^{\alpha}=O_{\delta}(1) \quad \text { and } \quad J_{i}^{\alpha}=O_{\delta}(\varepsilon) \quad \text { if } \quad i \neq i_{0}
$$




\section{Proof of Lemma 4.2}

The computation of the space derivatives for $i=i_{0}$ are straightforward. For $i \neq i_{0}$, the estimates like $O_{\delta}(1)$ of the space derivatives $\partial_{p} \Psi_{i}^{\alpha}$ for $p=1,2$ and $\partial_{11} \Psi_{i}^{\alpha}$ follow from assumption (A).

We have

$$
\partial_{t} \Psi_{i}^{\alpha}=\left(-\frac{\gamma_{i} X_{i}^{\prime}}{\varepsilon}+\frac{\gamma_{i}^{\prime}}{\gamma_{i}} \xi_{i}^{1}\right) \partial_{1} \psi_{i}^{\alpha}
$$

and

$$
\partial_{t t} \Psi_{i}^{\alpha}=\left(-\frac{\gamma_{i} X_{i}^{\prime}}{\varepsilon}+\frac{\gamma_{i}^{\prime}}{\gamma_{i}} \xi_{i}^{1}\right)^{2} \partial_{11} \psi_{i}^{\alpha}+\left[-\frac{\left(\gamma_{i} X_{i}^{\prime}\right)^{\prime}}{\varepsilon}+\left(\frac{\gamma_{i}^{\prime}}{\gamma_{i}}\right)^{\prime} \xi_{i}^{1}+\frac{\gamma_{i}^{\prime}}{\gamma_{i}}\left\{-\frac{\gamma_{i} X_{i}^{\prime}}{\varepsilon}+\frac{\gamma_{i}^{\prime}}{\gamma_{i}} \xi_{i}^{1}\right\}\right] \partial_{1} \psi_{i}^{\alpha} .
$$

We first notice that the second line of (1.14) implies that $\gamma_{i}$ is bounded, and then $\gamma_{i}^{\prime}$ is also bounded as a consequence of (1.6). Using again assumptions (A), we immediately obtain the desired estimates for $\partial_{t} \Psi_{i}^{\alpha}$ and $\partial_{t t} \Psi_{i}^{\alpha}$ in each case $i=i_{0}$ and $i \neq i_{0}$.

\section{Proof of Theorem 1.1}

The main result of this section is Proposition 5.1 below which will imply Theorem 1.1.

\section{Proposition 5.1 (Plugging the ansatz in the equations)}

Let $\hat{u}^{\varepsilon}$ given by (1.5) with $v^{\varepsilon}$ defined in (1.7) for general coefficients $a_{i}^{\alpha}(t)$ and for $\phi$ solution of (1.3) and for general $\psi^{\alpha}, \alpha=1,2,3$ such that assumption (A) holds. We assume moreover that

$$
\left|\Delta \psi^{\alpha}(x)\right| \leq \frac{C}{1+\left|x_{1}\right|} \quad \text { for } \quad \alpha=1,2,3 .
$$

We assume (1.14) and also that $W, \sigma$ are smooth enough. Then for any $(x, t) \in \mathbb{R} \times \mathbb{R}^{+} \times[0, T]$, we have the following estimates with the index $i_{0}=i_{0}\left(x_{1}, t\right)$ defined in Lemma 4.1:

$$
\left\{\begin{array}{l}
\square \hat{u}^{\varepsilon}=O_{\delta}(1)+\frac{1}{\varepsilon_{1}} I_{i_{0}}^{1} \\
N_{\varepsilon} \hat{u}^{\varepsilon}=O_{\delta}(1)+\frac{1}{\varepsilon} I_{i_{0}}^{2}
\end{array}\right.
$$

where

$$
\left\{\begin{aligned}
I_{i}^{1}= & -\frac{1}{c_{0}^{2}}\left\{2 \gamma_{i}^{\prime} X_{i}^{\prime} \xi_{i}^{1} \partial_{11} \phi_{i}+\left(\gamma_{i}^{\prime} X_{i}^{\prime}+\left(\gamma_{i} X_{i}^{\prime}\right)^{\prime}\right) \partial_{1} \phi_{i}\right\}+\sum_{\alpha=1,2,3} a_{i}^{\alpha}(t) \Delta \psi_{i}^{\alpha}, \\
I_{i}^{2}= & \frac{1}{\alpha_{0}}\left(-\sigma\left(X_{i}, t\right)+\frac{1}{\pi} \sum_{j \neq i} \frac{1}{\gamma_{j}\left(X_{i}-X_{j}\right)}\right)\left(W^{\prime \prime}(0)-W^{\prime \prime}\left(\phi_{i}\right)\right) \\
& -\left\{2 m \gamma_{i}^{\prime} X_{i}^{\prime} \xi_{i}^{1} \partial_{11} \phi_{i}+\left\{k \gamma_{i} X_{i}^{\prime}+m\left(\gamma_{i}^{\prime} X_{i}^{\prime}+\left(\gamma_{i} X_{i}^{\prime}\right)^{\prime}\right)\right\} \partial_{1} \phi_{i}\right\}+\sum_{\alpha=1,2,3} a_{i}^{\alpha}(t) A \psi_{i}^{\alpha} .
\end{aligned}\right.
$$

with

$$
\left\{\begin{array}{l}
\Delta \psi_{i}^{\alpha}:=\partial_{11} \psi_{i}^{\alpha}+\partial_{22} \psi_{i}^{\alpha} \\
A \psi_{i}^{\alpha}=\beta \partial_{11} \psi_{i}^{\alpha}+\partial_{2} \psi_{i}^{\alpha}-W^{\prime \prime}\left(\phi_{i}\right) \psi_{i}^{\alpha}
\end{array}\right.
$$




\section{Proof of Theorem 1.1}

Fom equations (1.9), (1.10) and (1.11) and assumption (A), we deduce that (5.1) holds. The same three equations also yield

$$
\begin{cases}\sum_{\alpha=1,2,3} a_{i}^{\alpha} \Delta \psi^{\alpha}=a_{i}^{1} x_{1} \partial_{11} \phi+a_{i}^{2} \partial_{1} \phi, & x_{2}>0 \\ \sum_{\alpha=1,2,3} a_{i}^{\alpha} A \psi^{\alpha}=\frac{f}{\alpha_{0}}\left(W^{\prime \prime}(\phi)-W^{\prime \prime}(0)\right)+\beta a_{i}^{1} x_{1} \partial_{11} \phi+\left(\beta a_{i}^{2}+a_{i}^{3}\right) \partial_{1} \phi, & x_{2}=0\end{cases}
$$

with

$$
f:=\bar{\beta}_{0}\left(-\frac{a_{i}^{1}}{2}+a_{i}^{2}\right)+\bar{k}_{0} a_{i}^{3} .
$$

Then we see that $I_{i}^{1}=0$ if and only if

$$
\left\{\begin{array}{l}
a_{i}^{1}=\frac{2 \gamma_{i}^{\prime} X_{i}}{c_{0}^{2}} \\
a_{i}^{2}=\frac{\gamma_{i}^{\prime} X_{i}^{\prime}+\left(\gamma_{i} X_{i}^{\prime}\right)^{\prime}}{c_{0}^{2}}
\end{array}\right.
$$

and $I_{i}^{2}=0$ if and only if

$$
\left\{\begin{array}{l}
f=-\sigma\left(X_{i}, t\right)+\frac{1}{\pi} \sum_{j \neq i} \frac{1}{\gamma_{j}\left(X_{i}-X_{j}\right)} \\
\beta a_{i}^{1}=2 m \gamma_{i}^{\prime} X_{i}^{\prime}, \\
\beta a_{i}^{2}+a_{i}^{3}=k \gamma_{i} X_{i}^{\prime}+m\left(\gamma_{i}^{\prime} X_{i}^{\prime}+\left(\gamma_{i} X_{i}^{\prime}\right)^{\prime}\right) .
\end{array}\right.
$$

We therefore see that (5.4) and (5.5) are satisfied if and only if $\beta=m c_{0}^{2}$, the coefficients $a_{i}^{\alpha}$ are given by (1.8) and the ODE dynamics (1.6) for the coefficients $m_{0}, k_{0}$ given by (1.13).

We now turn to the proof of Proposition 5.1.

\section{Proof of Proposition 5.1}

The proof is made in several steps.

\section{Step 1: computation of $\square \hat{u}^{\varepsilon}$}

Using Lemma 4.2 (precisely we use (4.6) and the fourth line of (4.5)), we get

$$
\square \hat{u}^{\varepsilon}=O_{\delta}(1)+\sum_{i}\left\{\frac{1}{\varepsilon^{2}}\left(A_{1} \phi_{i}+\varepsilon \frac{J_{i}^{0}}{c_{0}^{2}}\right)-\frac{1}{\varepsilon}\left\{\sum_{\alpha=1,2,3} a_{i}^{\alpha}(t)\left(A_{1} \psi_{i}^{\alpha}+\varepsilon \frac{J_{i}^{\alpha}}{c_{0}^{2}}\right)\right\}\right\},
$$

where for $\alpha=0,1,2,3$

$$
A_{1} \psi_{i}^{\alpha}:=\left\{\left(\frac{\gamma_{i} X_{i}^{\prime}}{c_{0}}\right)^{2} \partial_{11} \psi_{i}^{\alpha}-\gamma_{i}^{2} \partial_{11} \psi_{i}^{\alpha}-\partial_{22} \psi_{i}^{\alpha}\right\}=-\Delta \psi_{i}^{\alpha}
$$


which reads explicitly for $\alpha=0$ :

$$
A_{1} \phi_{i}=-\Delta \phi_{i}=0
$$

Using moreover (4.7) and (5.1), we get

$$
\square \hat{u}^{\varepsilon}=O_{\delta}(1)+\frac{1}{\varepsilon}\left\{\frac{J_{i_{0}}^{0}}{c_{0}^{2}}-\sum_{\alpha=1,2,3} a_{i_{0}}^{\alpha}(t) A_{1} \psi_{i_{0}}^{\alpha}\right\}=O_{\delta}(1)+\frac{1}{\varepsilon} I_{i_{0}}^{1} .
$$

\section{Step 2: computation of $N_{\varepsilon} \hat{u}^{\varepsilon}$}

\section{Step 2.1: computation}

Using Lemma 4.2, we get

$$
\begin{aligned}
N_{\varepsilon} \hat{u}^{\varepsilon}= & O_{\delta}(1)+\frac{W^{\prime}\left(\hat{u}^{\varepsilon}\right)}{\varepsilon^{2}}-\frac{\sigma}{\varepsilon} \\
& +\sum_{i}\left\{\frac{1}{\varepsilon^{2}}\left(A_{2} \phi_{i}+\varepsilon m J_{i}^{0}-\varepsilon k \gamma_{i} X_{i}^{\prime} \partial_{1} \phi_{i}\right)-\frac{1}{\varepsilon} \sum_{\alpha=1,2,3} a_{i}^{\alpha}\left(A_{2} \psi_{i}^{\alpha}+\varepsilon m J_{i}^{\alpha}-\varepsilon k \gamma_{i} X_{i}^{\prime} \partial_{1} \psi_{i}^{\alpha}\right)\right\}
\end{aligned}
$$

with for $\alpha=0,1,2,3$

$$
A_{2} \psi_{i}^{\alpha}:=\beta\left(\frac{\left(\gamma_{i} X_{i}^{\prime}\right)^{2}}{c_{0}^{2}} \partial_{11} \psi_{i}^{\alpha}-\gamma_{i}^{2} \partial_{11} \psi_{i}^{\alpha}\right)-\partial_{2} \psi_{i}^{\alpha}=-\beta \partial_{11} \psi_{i}^{\alpha}-\partial_{2} \psi_{i}^{\alpha} .
$$

Using (4.7) and assumption (A), we deduce

$$
N_{\varepsilon} \hat{u}^{\varepsilon}=O_{\delta}(1)+\frac{1}{\varepsilon^{2}}\left(W^{\prime}\left(\hat{u}^{\varepsilon}\right)+A_{2} \phi_{i_{0}}\right)+\frac{1}{\varepsilon}\left\{-\sigma+\frac{1}{\varepsilon} \sum_{i \neq i_{0}} A_{2} \phi_{i}+m J_{i_{0}}^{0}-k \gamma_{i_{0}} X_{i_{0}}^{\prime} \partial_{1} \phi_{i_{0}}-\sum_{\alpha=1,2,3} a_{i_{0}}^{\alpha} A_{2} \psi_{i_{0}}^{\alpha}\right\} .
$$

From equation (1.3) we have, for all $i=1, \ldots, N$

$$
A_{2} \phi_{i}=-W^{\prime}\left(\phi_{i}\right)=-W^{\prime}\left(\tilde{\phi}_{i}\right) .
$$

From assumption (A) we deduce that for $i \neq i_{0}$,

$$
A_{2} \phi_{i}=-W^{\prime \prime}(0) \tilde{\phi}_{i}+O\left(\tilde{\phi}_{i}^{2}\right)=\frac{1}{\pi \xi_{i}^{1}}+O_{\delta}\left(\varepsilon^{2}\right),
$$

then

$$
N_{\varepsilon} \hat{u}^{\varepsilon}=O_{\delta}(1)+\frac{1}{\varepsilon^{2}}\left(W^{\prime}\left(\hat{u}^{\varepsilon}\right)-W^{\prime}\left(\tilde{\phi}_{i_{0}}\right)\right)+\frac{1}{\varepsilon}\left\{-\sigma+\sum_{i \neq i_{0}} \frac{1}{\pi \varepsilon \xi_{i}^{1}}+m J_{i_{0}}^{0}-k \gamma_{i_{0}} X_{i_{0}}^{\prime} \partial_{1} \phi_{i_{0}}-\sum_{\alpha=1,2,3} a_{i_{0}}^{\alpha} A_{2} \psi_{i_{0}}^{\alpha}\right\} .
$$

Using now Lemma 4.1, we get

$$
N_{\varepsilon} \hat{u}^{\varepsilon}=O_{\delta}(1)+\frac{1}{\varepsilon}\left\{\begin{array}{l}
W^{\prime \prime}\left(\tilde{\phi}_{i_{0}}\right)\left\{\frac{\sigma}{\alpha_{0}}-\sum_{\alpha=1,2,3} a_{i_{0}}^{\alpha} \psi_{i_{0}}^{\alpha}-\sum_{i \neq i_{0}} \frac{1}{\alpha_{0} \pi \varepsilon \xi_{i}^{1}}\right\}+\sum_{i \neq i_{0}} \frac{1}{\pi \varepsilon \xi_{i}^{1}} \\
-\sigma+m J_{i_{0}}^{0}-k \gamma_{i_{0}} X_{i_{0}}^{\prime} \partial_{1} \phi_{i_{0}}-\sum_{\alpha=1,2,3} a_{i_{0}}^{\alpha} A_{2} \psi_{i_{0}}^{\alpha}
\end{array}\right\} .
$$


Using the fact that

$$
A_{2} \psi_{i_{0}}^{\alpha}=-A \psi_{i_{0}}^{\alpha}-W^{\prime \prime}\left(\phi_{i_{0}}\right) \psi_{i_{0}}^{\alpha}
$$

we get

$$
N_{\varepsilon} \hat{u}^{\varepsilon}=O_{\delta}(1)+\frac{1}{\varepsilon}\left\{\begin{array}{c}
\left(W^{\prime \prime}\left(\tilde{\phi}_{i_{0}}\right)-W^{\prime \prime}(0)\right)\left\{\frac{\sigma}{\alpha_{0}}-\sum_{i \neq i_{0}} \frac{1}{\alpha_{0} \pi \varepsilon \xi_{i}^{1}}\right\} \\
+m J_{i_{0}}^{0}-k \gamma_{i_{0}} X_{i_{0}}^{\prime} \partial_{1} \phi_{i_{0}}+\sum_{\alpha=1,2,3} a_{i_{0}}^{\alpha} A \psi_{i_{0}}^{\alpha}
\end{array}\right\}
$$

\section{Step 2.2: evaluation}

We now write $\varepsilon \xi_{i}^{1}=\gamma_{i}\left(X_{i_{0}}-X_{i}\right)+\frac{\gamma_{i}}{\gamma_{i_{0}}} \varepsilon \xi_{i_{0}}^{1}$ to obtain for $i \neq i_{0}$ :

$$
\frac{1}{\alpha_{0} \pi \varepsilon \xi_{i}^{1}}=\frac{1}{\alpha_{0} \pi \gamma_{i}\left(X_{i_{0}}-X_{i}\right)}+O_{\delta}\left(\varepsilon \xi_{i_{0}}^{1}\right)
$$

where we have used assumption (1.14). Therefore

$$
\frac{1}{\varepsilon}\left(W^{\prime \prime}\left(\tilde{\phi}_{i_{0}}\right)-W^{\prime \prime}(0)\right) \sum_{i \neq i_{0}} \frac{1}{\alpha_{0} \pi \varepsilon \xi_{i}^{1}}=\frac{1}{\varepsilon}\left(W^{\prime \prime}\left(\tilde{\phi}_{i_{0}}\right)-W^{\prime \prime}(0)\right) \sum_{i \neq i_{0}} \frac{1}{\alpha_{0} \pi \gamma_{i}\left(X_{i_{0}}-X_{i}\right)}+O_{\delta}\left(\xi_{i_{0}}^{1} \tilde{\phi}_{i_{0}}\right) .
$$

Finally, by plugging this relation into (5.6) and using assumption (A) to see that $O_{\delta}\left(\xi_{i_{0}}^{1} \tilde{\phi}_{i_{0}}\right)=$ $O_{\delta}(1)$, we obtain the second equation of (5.2).

\section{References}

[1] X. CABré And J. SolÀ-Morales Layer solutions in a half-space for boundary reactions, Comm. Pure Appl. Math., 58 (12): 1678-1732, 2005.

[2] C. Denoual, Dynamic dislocation modeling by combining Peierls-Nabarro and Galerkin methods, Physical Review B 70, 024106 (2004).

[3] C. Denoual, Modeling dislocation by coupling Peierls-Nabarro and element-free Galerkin methods, Comput. Methods Appl. Mech. Engrg. 196 (2007) 1915-192.

[4] A. El Haju, H. Ibrahim and R. Monneau, Dislocation dynamics: from microscopic models to macroscopic crystal plasticity, Continuum Mech. Thermodyn. (2009) 21: 109123.

[5] J. D. Eshelby, Uniformly moving dislocations, Proc. Phys. Soc. A, 62, 307-314.

[6] A. Fino, H. Ibrahim and R. Monneau, The Peierls-Nabarro model as a limit of a FrenkelKontorova model, J. Differential Equations 252 (2012) 258-293.

[7] M. Gonzalez And R. Monneau, Slow motion of particle systems as a limit of a reactiondiffusion equation with half-Laplacian in dimension one, DCDS-A 32 (4) (2012), 1255-1286.

[8] J. R. Hirth And L. Lothe, Theory of dislocations, Second Edition. Malabar, Florida: Krieger, 1992. 
[9] J. R. Hirth, H. M. ZBib And L. Lothe, Forces on high velocity dislocations, Model. Simul. Mater. Sci. Eng, 6 (2), 165-169.

[10] R. Monneau And S. Patrizi, Homogenization of the Peierls-Nabarro model for dislocation dynamics, J. Differential Equations 253 (7) (2012), 2064-2105.

[11] F. R. N. Nabarro, Fifty-year study of the Peierls-Nabarro stress, Material Science and Engineering A 234-236, p. 67-76, 1997.

[12] Y. P. Pellegrini, Dynamic Peierls-Nabarro equations for elastically isotropic crystals, Phys. Rev. B 81, 024101 (2010).

[13] L. Pillon, Modélisations du mouvement instationnaire et des interactions de dislocations, $\mathrm{PhD}$ thesis, Pierre et Marie Curie University, Paris, France, (2008).

[14] L. Pillon and C. Denoual, Inertial and retardation effects for dislocation interactions, Philosophical Magazine - Vol. 89 - Issue 2 - 2009 - pp. 127-141.

[15] L. Pillon, C. Denoual, R. Madec and Y. P. Pellegrini, Influence of inertia on the formation of dislocation dipoles, Journal de Physique IV (Proceedings), Volume 134, Issue 1, August 2006, pp.49-54.

[16] L. Pillon, C. Denoual and Y. P. Pellegrini, Equation of motion for dislocations with inertial effects, Phys. Rev. B 76, 224105 (2007). 